\title{
Introducing a Micro-wireless Architecture for Business Activity Sensing
}

\author{
Raj Bridgelall, Senior Member, IEEE
}

\begin{abstract}
RFID performance deficiencies discovered in recent high profile applications have highlighted the danger of selecting only passive tags for an application because of their lowest cost relative to other types of RFID tags. Consequentially, battery-based RFID technologies are being considered to fill those performance gaps. A mix of both passive and battery-based RFID technologies can provide a more cost effective and robust solution than a homogeneous RFID deployment. However, it is easy to choose the wrong battery-based RFID technology given the confusing array of choices currently available. This paper explores the performance deficiencies of both passive and battery-based RFID technologies. A new micro-wireless technology that resolves these performance deficiencies is then introduced. Finally an application example is presented that demonstrates how the new technology can also seamlessly roam between passive and battery-based RFID infrastructures at the lowest possible cost to bridge their respective performance gaps.
\end{abstract}

Index Terms-Active RFID, Battery Assisted Passive (BAP), Far-field, Near-field, Passive RFID, Wireless Sensors

\section{INTRODUCTION}

$\mathrm{T}$ HIS paper provides an overview of the three fundamental RFID architectures in use today: passive, battery assisted passive (BAP), and active. Passive RFID tags do not carry their own energy source. They operate by harvesting energy from the reader, and send data by reflecting energy back to the reader. Active RFID operates by utilizing energy from a battery or an equivalent local energy source. They send data to a reader by producing a low-power modulated signal. BAP RFID is a hybrid architecture that sends data by reflecting energy from the reader in the same manner as passive RFID, but utilizes a battery for its overall operation.

Each type of architecture communicates either in the nearfield or the far-field. The most popular near-field systems communicate at low frequencies (LF) such as $125 \mathrm{kHz}$ and at high frequencies (HF) such as $13.56 \mathrm{MHz}$ using magnetic field coupling rather than electric field propagation.

Following this overview is a more in-depth discussion of each type of RFID architecture. A new category is then introduced that combines the benefits of each of the previous

Manuscript received December 19, 2007.

R. Bridgelall, Vice President \& CTO for Axcess International, Inc., 3208 Commander Drive, Carrollton, TX 75006 USA (972-250-5967);

e-mail: raj.bridgelall@ieee.org. architectures in a manner that enables existing application requirements to be more readily addressed. This new architecture also enables applications that were not previously possible.

\section{A. Passive RFID}

Passive RFID systems have improved substantially since the introduction of first generation of ultra high frequency (UHF) systems. Improvements in range and interoperability, multi-tag arbitration speed, and interference susceptibility were promised and delivered with the ratification of EPC Class I Generation 2 (C1G2) and the ISO 18000-6 standards [1]. Although passive UHF RFID performance enhancements, cost reduction, and end-user mandates helped to improve the technology adoption rate, the level of deployment has yet to meet industry expectations. A dominant reason for the slow adoption rate of RFID is a mismatch in expectations between technological capabilities and application requirements. The passive tag's read rates, localization accuracy, interference resilience, infrastructure simplicity and maintenance costs have not met the needs of several high profile applications [2], [3].

\section{B. Battery Assisted Passive RFID}

Although further improvements in passive RFID technologies are inevitable, the degree of improvement using traditional approaches will be limited [4]. For example, adding a battery to improve a tag's receiving sensitivity and avoid remote power transfer from the reader does improve omni-directional range, but only to a modest extent [5]. We will see that utilizing a battery for a tag to transmit power instead of reflecting or backscattering power will provide a relatively large magnitude of improvement in omnidirectional range especially with small antennas. Batteryassisted passive tags are also sometimes called semi-passive tags.

\section{Active RFID}

A tag that utilizes the battery to emit rather than reflect or backscatter RF energy is often called an active tag. Although a battery can substantially improve performance, it will also limit maintenance-free operational life. Harvesting energy from sources such as vibration or light has been shown to address this shortcoming but these sources must be adequate and available throughout the life of the application [6]. Section II explores the performance 
limitations of passive and semi-passive backscattering RFID.

Active tags substantially improve range and read rate in electromagnetically unfriendly environments. Greater range improves the link quality in most applications but also exacerbates the problems of RF interference and position determination. Systems that propagate RF energy produce signal reflections that cause multipath interference. This makes it difficult to pinpoint a tag's location and provide for directionality of movement, even when using sophisticated computational techniques [7]. UHF RFID systems, whether active, passive or semi-passive propagate a signal in the farfield that can also generate interference for nearby readers and tags [8]. Some active RF propagation systems such as Wi-Fi, ZigBee, and Bluetooth incorporate complex protocols to reduce the impact from multi-path interference, but the trade-off is increased power consumption [9], [10], [11]. Complex air-interface protocols will require larger and more expensive batteries than a simpler protocol. These and other performance limitations of active RFID will be explored in Section III.

\section{Dual-active RFID}

Passive and battery-based RFID systems communicate in either the near-field or the far-field. Passive tags generally transmit a signal to a reader via some form of signal reflection while battery-based systems tend to utilize radiated emissions. Near-field RFID operates by magnetic field induction between the reader and the tag. The reader behaves like a primary coil and the tag like a secondary coil of a loosely coupled transformer. Near-field RFID standards typically require operation at either a low-frequency such as $125 \mathrm{kHz}$, or a high-frequency such as $13.56 \mathrm{MHz}$ [12], [13]. Near-field antennas create well controlled read zones, for example around a portal, and provide a degree of covertness. The magnetic field, especially at LF, penetrates dense RF media and does not reflect in a manner that produces a multipath interference problem. The magnetic field provides for robust localization, but unlike far-field propagation, it cannot radiate energy across relatively long distances. Practical RFID solutions require both robust localization and reliable long distance communications. Hence, a hybrid solution that incorporates both near-field and far-field capabilities is needed. Section IV introduces this new category called dual-active RFID and describes how difficult application requirements can now be more cost-effectively addressed.

\section{E. Dual-active micro-wireless}

The lower cost of passive tags is a dominant reason for their initial consideration in most RFID applications. After a few pilot projects and analysis, it is often realized that a portion of the application will require the link robustness and reliability that active tags can provide. Additionally, unplanned business process changes are often incurred in order to make up for passive RFID performance deficiencies. For example, a separate process to sort items has become necessary for many consumer product manufacturers in order to ensure that passive tags are placed on specific areas of an item, and affixed in an orientation that minimizes radiated energy gaps. Sometimes, more drastic measures are taken such redesigning the package with more air gaps to let RF energy more easily reach passive tags [14]. Active RFID tags are generally more capable of overcoming these deficiencies and, so are easier to integrate into an existing business process.

Recognizing that both passive and battery-based RFID architectures have particular strengths and weaknesses, some advanced end-users have begun to utilize a technological mix in an effort to create a complete solution set. A heterogeneous deployment that utilizes passive, BAP, and active RFID tags can improve read rates, minimize business process changes, and significantly reduce related adoption barriers. However, this approach is still not optimal because of the added infrastructure complexity and cost for managing disparate and incompatible technologies.

Section V describes a new dual-active micro-wireless sensor chip called the Enterprise Dot ${ }^{\mathrm{TM}}$. The technology used includes both backscatter and active far-field communication modes for maximum agility when roaming across incompatible passive and active RFID infrastructures. The backscatter UHF capability enables communications in any passive UHF RFID infrastructure and can, therefore, easily bridge the gap between low-cost passive and robust active RFID performance.

Section VI describes a cold-chain application that benefits from this new ability to seamlessly roam across numerous wireless infrastructures. Section VII concludes the paper.

\section{BACKSCATTER SYSTEM LIMITATIONS}

Backscatter tags transmit data by "reflecting" the continuous wave $(\mathrm{CW})$ energy received from a reader. The rate of change between energy absorption and reflection states encodes the bits. For example, the reader will interpret the time between magnitude and/or phase changes of the reflected energy as information bits. Hence, this type of communications is sometimes referred to as modulated backscatter transmission. Passive backscatter tags harvest energy from the reader to power their circuits. It is also possible for tags to harvest energy from other sources such as vibration and light. BAP tags utilize a battery to power their circuits instead of harvesting RF energy.

Given regulatory power and bandwidth limitations, the link margin between the reader and the backscatter tag is constrained by either tag receiver sensitivity or reader receiver sensitivity. The higher the tag receiver sensitivity, the weaker the signal, and the further away it can reliably decode reader commands. The reader's transmitted power diminishes at the rate of $\left(1 / \mathrm{r}^{2}\right)$ in the unobstructed far-field where $\mathbf{r}$ is the separation distance between the reader and tag antennas. The tag receives power as given by equation (1),

$$
P_{\text {tag_rx }}=\psi_{\mathrm{r}}\left(1 / \mathrm{r}^{2}\right)
$$


The parameter $\psi_{\mathrm{r}}$ is proportional to the reader transmitted power and the carrier wavelength [4]. In particular, the power received is a function of the spatially distributed antenna radiation pattern. The maximum power received is located along the spatial trajectory where $\psi_{\mathrm{r}}=\left[\mathrm{P}_{\mathrm{tx}}\right] \lambda^{2} /(4 \pi)^{2}$. $\mathrm{P}_{\mathrm{tx}}$ is the effective reader radiated power in Watts. It is important to note that this expression is accurate only in the far-field. The near-field/far-field boundary is typically defined as a distance from the antenna where the wave impedance quickly approaches the free-space impedance value of $377 \mathrm{ohms}$ [15]. The near-field radius is a function of the carrier wavelength, $\lambda$ and is given by equation (2),

$$
\mathrm{R}_{\mathrm{NF}}=\lambda /(2 \pi)
$$

For most practical far-field UHF RFID systems, this distance is less than one foot. Upon decoding the reader command, the tag backscatter modulates a response. Some of the energy incident on the tag's antenna will be lost to impedance boundary absorption. Therefore, the propagating reader signal is further weakened before it is reflected from the tag. In addition to these absorption losses, the signal undergoes a return path loss that is identical to the forward link path loss. Therefore, the total backscatter signal loss will be a function of $\left(1 / \mathrm{r}^{4}\right)$ as given by equation (3).

$$
P_{\text {reader_rx }}=\Psi_{t}\left(1 / r^{4}\right)
$$

The parameter $\Psi_{\mathbf{t}}$ includes the forward path loss factors from $\psi_{\mathbf{r}}$ as well as losses from energy absorbed by the tag [5]. A tag reader can decode the reflected signal if it has adequate receiver sensitivity. For most commercially available systems, passive tag range is typically limited by tag receiver sensitivity rather than reader receiver sensitivity. This is due a design trade-off between optimizing for maximum RF energy harvesting and optimizing for maximum receiver sensitivity. Since RF energy harvesting is not a requirement for semi-passive tags, their designs can be optimized for maximum receiver sensitivity. Consequently, the reader receiver sensitivity typically becomes the limiting factor for semi-passive RFID range. Reader receiver sensitivity is in turn limited by its architecture, which is constrained by the RADAR backscatter problem of isolating a transmitted signal from a co-located receiver [16].

These technical constraints in receiver sensitivity adversely limit the far-field backscatter RFID link margin. Tags are generally required to operate in electromagnetically unfriendly environments consisting of liquids and metals. Liquids absorb RF energy. Metals contribute to multi-path reflections that can cause signal cancellation when reflected signal components combine at the receiver in anti-phase. While the free-space path loss for backscatter RFID systems is of $\left(1 / \mathrm{r}^{4}\right)$, an RF absorptive and reflective environment can contribute an even greater signal loss of $\left(1 / \mathrm{r}^{12}\right)$ [17].

\section{ACTIVE SySTEM LiMITATIONS}

Active tags can operate in the near-field or the far-field. This section focuses on issues relevant to the most popular long-range UHF systems that operate in the far-field.

\section{A. Multi-path Environments}

Active far-field tags transmit a signal by emitting energy transformed from its power source rather than reflecting incident energy from the reader. A typical active tag can transmit orders of magnitude more power than a backscatter tag will reflect at the same distance. For example, a $4 \mathrm{~W}$ $(\sim 36 \mathrm{dBm})$ signal transmitted from a passive RFID reader at $915 \mathrm{MHz}$ will become approximately 3 microwatts $(-25.36$ $\mathrm{dBm}$ ) after traveling a distance of 100 feet in free-space. This is with respect to the maximum signal level legally allowed in North America for transmission in the unlicensed $915 \mathrm{MHz}$ frequency band [18]. A backscatter tag, whether battery assisted or not, reflects a portion of this signal to the passive tag reader. A low power active tag in the same band will typically transmit a signal at $1 \mathrm{~mW}$. The radiated signal power from the active tag is over 300 times greater than that of the backscatter signal power reflected from a passive tag. Thus a $915 \mathrm{MHz}$ reader antenna will receive an active tag signal from 100-feet away that is over 300 times greater. A stronger signal improves a tag's chances of being received in any application.

Although this improved link margin can result in higher read rates, the stronger signal can also degrade tag localization accuracy. Multi-path interference adversely affects far-field passive, semi-passive, and active RFID

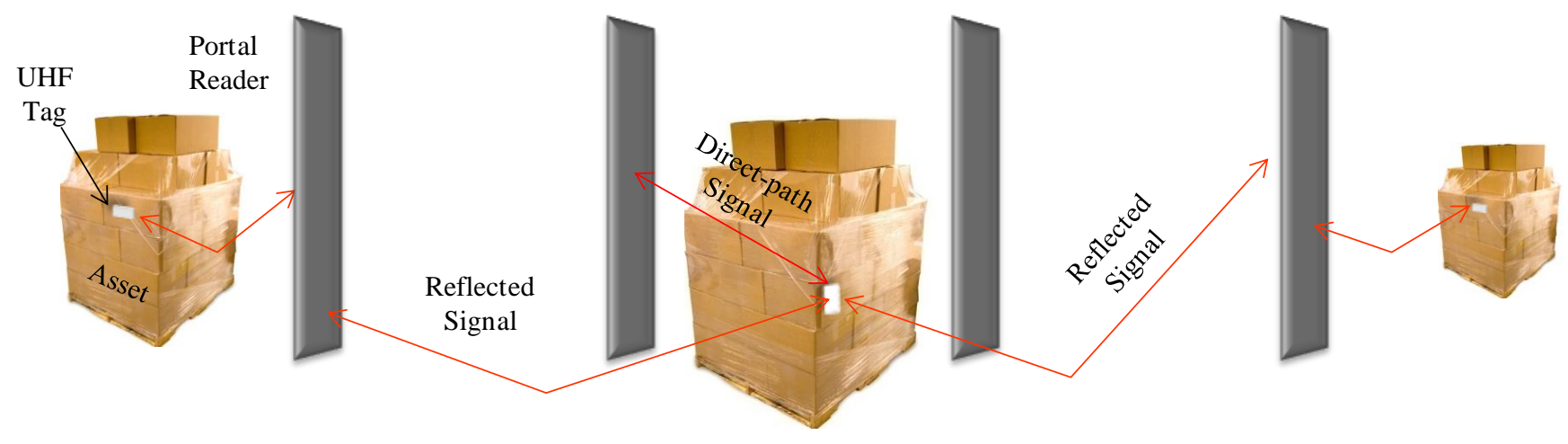

Fig. 1. Configuration of typical portal readers. 


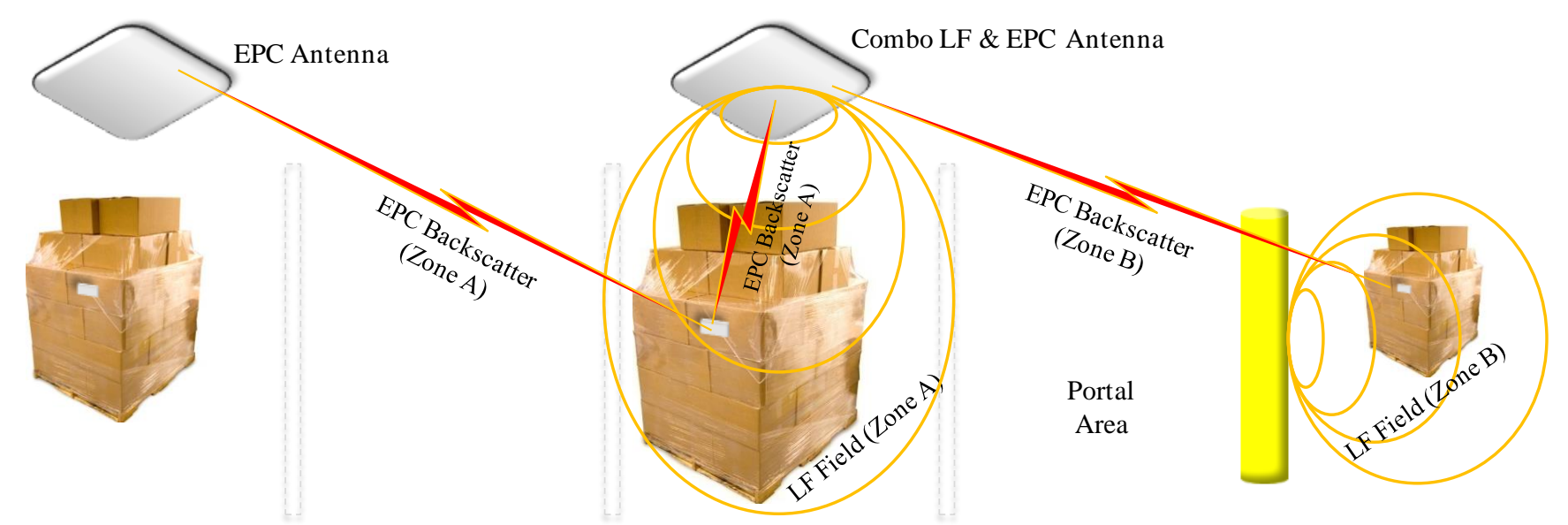

Fig. 2. Dual-active portal reader configuration.

systems in the same manner. Zone control and tag directionality is compromised when signals reflect uncontrollably in a highly cluttered environment. Attempts to increase range by radiating a stronger signal only exacerbates this problem [16].

Fig. 1 illustrates the zone control problem for a multiportal installation such as the dock doors in a warehouse. While the improved link margin delivers more robust omnidirectional reading and larger read zones, it becomes increasingly difficult to localize the read zones. At first glance, one might consider filtering out signals by their received signal strength and then applying some sort of signal correlation across multiple readers. However, a reflected but indirect path signal can be stronger than an obstructed direct path signal. For example, a direct path signal may be so heavily attenuated when it passes through RF absorptive items that it becomes much weaker than a signal reflected from the ground or a piece of metallic equipment. Signal strength variability from item absorption, reflections from moving objects, and variations in tag orientation also adds to signal inconsistencies. Therefore, signal strength alone cannot provide the information needed to discern shorter signal paths from the longer ones.

Shielding each portal zone may help but such a solution can be expensive, obtrusive, and can adversely constrain the normal workflow. Reducing signal strength may also help under certain circumstances but doing so adversely reduces link margin. Directional antennas can help to confine the radiated energy, but they are typically bulky and expensive.

The direction of tag travel through a portal is also often required. It is possible to utilize computational positioning techniques to determine directionality to some degree of accuracy. This requires an ability to capture a tag's backscatter signal for a sufficiently long period of time and with a high enough sample rate to reduce the errors from multi-path propagation. Such a requirement can burden the application by limiting multi-tag detection rates, constraining the item transport speed, and increasing the complexity and cost of readers. A dual-active RFID system with near-field zone control in each portal can overcome this problem. A cost effective solution using dual-active tags is described in Section IV.

\section{B. Battery Life}

In previous sections, we've shown that battery powered RFID systems deliver significantly improved performance over passive RFID systems, and minimizes upfront business process changes needed to accommodate its implementation. Although a battery adds initial material cost over passive solutions, the savings accumulated from "soft" benefits could compensate for this added expense over the life of the tagged item. For many applications, these soft benefits include improved data capture reliability, greater asset visibility, ease of deployment, and negligible upfront business process adjustments. In general, the longer the battery life, the lower the amortized cost of an active tag over the life of a tagged item.

Given a tag's battery, its useful life is a function of both the tag's sleep mode power consumption and activation duty cycle. Power consumption depends on the electronic architecture, anti-collision protocol, and power management schemes. The architecture complexity is primarily determined from the wireless standard that a tag is designed to support. For example, Wi-Fi tags must be compatible with the IEEE 802.11 series of standards that were developed for high-speed wireless Internet applications. ISO 18000-7 tags are designed for operation within a less complex narrow-band channel at $433.92 \mathrm{MHz}$ [19]. In general, a lower complexity protocol will require fewer transistors to implement the hardware and also fewer processor instructions to complete the information exchange, thereby consuming less power.

Thin-film and printed battery technologies promise to dramatically lower the cost of active RFID technologies because they will be compatible with high volume roll-to-roll electronic manufacturing assembly processes. However, these emerging battery technologies cannot yet provide the energy densities of standard "coin-cell" or cylindrical 
batteries [20]. Low power consumption architectures that implement low-complexity RFID protocols can best leverage this early stage low-capacity battery technology to provide many months of maintenance-free operation.

\section{DUAL-ACTIVE RFID}

Previous sections described two of the most prominent active RFID issues which are zone localization in high multipath environments, and battery life. This section describes a merged architecture that adds near-field communications for both localization and wake-up power management, while retaining the distance propagation benefits of far-field communications.

\section{A. Control Point Architecture}

Dual-active RFID tags utilize both near-field and far-field communications so as to eliminate the deficiencies of each while harnessing the benefits of both [21]. Tag localization is achieved by generating a well controlled magnetic nearfield around each portal as shown in Fig. 2. An LF nearfield generator creates a unique activation zone by modulating a location identification (LID) signal that the dual-active tag can decode once it is activated in the zone. The tag then broadcasts the LID plus its unique tag identification (TID) number to a remote reader via UHF farfield communications.

The magnetic field's volume can be controlled to a high degree of accuracy by adaptively adjusting the intensity of the current in a coil antenna. This feature eliminates signal spill-over into areas outside of the activation zone. Only those dual-active tags that enter the zone will awaken from an energy conservation state to capture the LID and broadcast their TID, or they can take some other predetermined action such as sending a special command to control a door lock or activate a remote camera [22]. For this reason, the above approach is often described as a control point.

Generated LF magnetic fields are preferred for control points because they robustly penetrate dense media and their activation volume can be easily adjusted. The LF near-field is resilient to detuning from materials such as liquids and metals. A control point also provides battery power management because location or condition information can be transmitted only when the tag enters the activation zone. Otherwise the tag will remain in an ultra-low power sleep state while awaiting near-field activation [23].

Fig. 2. further illustrates how LF zone control solves the adjacent portal problem introduced in Section III. Each dock door is equipped with a low-cost near-field antenna that defines an activation zone around the portal. Near-field antennas may be located wherever the magnetic field can be extended to cover the area of interest. For example, antennas can be buried under concrete, hung from the ceiling, or installed around a door frame. As illustrated, the UHF antenna location and orientation is not critical since the activation and read zone is defined only by the magnetic field generated from the near-field antenna. The UHF antenna can be installed in a location within range and hidden from view. Alternatively, the near-field antenna may be combined with UHF antennas already installed within the portal. Since the location and orientation of the UHF antenna is not used for localization, a single UHF antenna can be shared amongst several near-field antennas.

A near-field control point also facilitates another important feature called functional linkage. When one or more items are activated in the same control zone, they can be treated as a collection of linked objects. For example, a person carrying an access control credential badge/tag can be automatically associated with the tagged assets in that zone. This association can be verified in a database that has stored the rules of a particular linkage. Functional linkage can be utilized to automatically verify that personnel are handling only those assets that they are authorized to handle. Applications include security, safety, and compliances with Sarbanes-Oxley and Occupational Safety and Health Administration (OSHA) legislation [24]. Functional linkage is not reliable with far-field only architectures because of the localization issues related to multi-path interference.

\section{B. Real-time Location System (RTLS)}

A near-field control point provides real-time positioning data by associating the LID with a physical or easily identifiable location, such as an office, a conference room, or an exit/entry portal. Directionality is provided by alternating between two near-field zones whereby one zone is located inside and the other outside the portal. Each LF antenna creates a separate but adjacent zone by modulating its respective field with a different LID. Speed is also determined from the TID timestamp difference as tags move through different zones [25]. Near-field LF generators are simple electronic devices that do not require network connectivity. They can be conveniently installed at strategic control points around the enterprise. Some versions switch between multiple LF antennas to provide instantaneous directionality information for tagged objects moving through a portal.

Alternative RTLS approaches require computational techniques called trilateration or triangulation. The former requires distance estimation between the tag and at least three spatially separated receivers that frame the entire volume to be monitored. Trilateration relies on distance measurements, which are typically estimated using received signal strength indication (RSSI) or time-difference-ofarrival (TDOA) techniques. Triangulation requires an estimate of the angle-of-arrival (AoA) signal between three or more receivers and the tag. Both computational positioning approaches require that the tag transmit at a sufficiently high rate and periodicity in order to provide enough samples for noise reduction and signal estimation. Regulatory bodies tend to significantly limit the transmission 
data rate and periodicity in some unlicensed frequency bands, and this makes solution variability using computational positioning techniques too high.

Distance or angle estimation is highly sensitive to multipath propagation and interference. Consequently, computational positioning techniques tend to achieve relatively poor tag location accuracy, especially indoors. In general, location estimation accuracy can be improved with more bandwidth, power, or spatial diversity. However, such designs also tend to significantly increase cost and size while reducing options for satisfying world-wide regulatory approvals.

A combination of high accuracy indoor near-field control points and outdoor computational positioning estimation techniques are desirable and effective. Dual-active RFID provides the best architecture to leverage the synergies of both approaches. For example, a control point near the exit of a building can automatically command an egress tag to begin transmitting with an appropriate periodicity that facilitates computational positioning when outside. When returning indoors, a control point command can disable or significantly slow down periodic transmissions to save energy and lengthen battery life. Eliminating unnecessary tag transmission will reduce RF traffic that may otherwise lead to interference and network congestion. While indoors, the tagged item can be located near the most recently reported control point. Therefore, the most appropriate locations for control points would be near choke-point areas such as doorways, hallways, and portals.

\section{THE ENTERPRISE DOT ${ }^{\mathrm{TM}}$}

Active RFID solutions will continue their cost reduction trend as chipsets become more widely available. Given similar costs and form-factors, chip-set differentiation will tend to focus on omni-directional range, multi-tag arbitration speed, and battery life. It has been discovered that even when a design achieves the optimum balance between range, speed, and battery life for one application it is not necessarily the solution that is preferred across all applications. Therefore, a solution that can adapt to an application's requirements is desirable. Domain knowledge tells us that an architecture based on software definable radio (SDR) techniques can meet the majority of these needs most of the time.

RFID and wireless technologies tend to utilize a single mode of operation and are consequently limited in their application scope. The chart of Fig. 3 summarizes the four fundamental RFID or wireless sensor categories and their operational characteristics in terms of key benefits and deficiencies. Each type utilizes one of several standards indicated. There are also numerous variations in operational characteristics within each category. For example, active far-field devices are available for both narrow-band and wide-band channels. Narrow-band RFID devices utilize tens of kilohertz of spectrum and typically operate in the globally available $433.92 \mathrm{MHz}$ band. Wide-band RFID typically

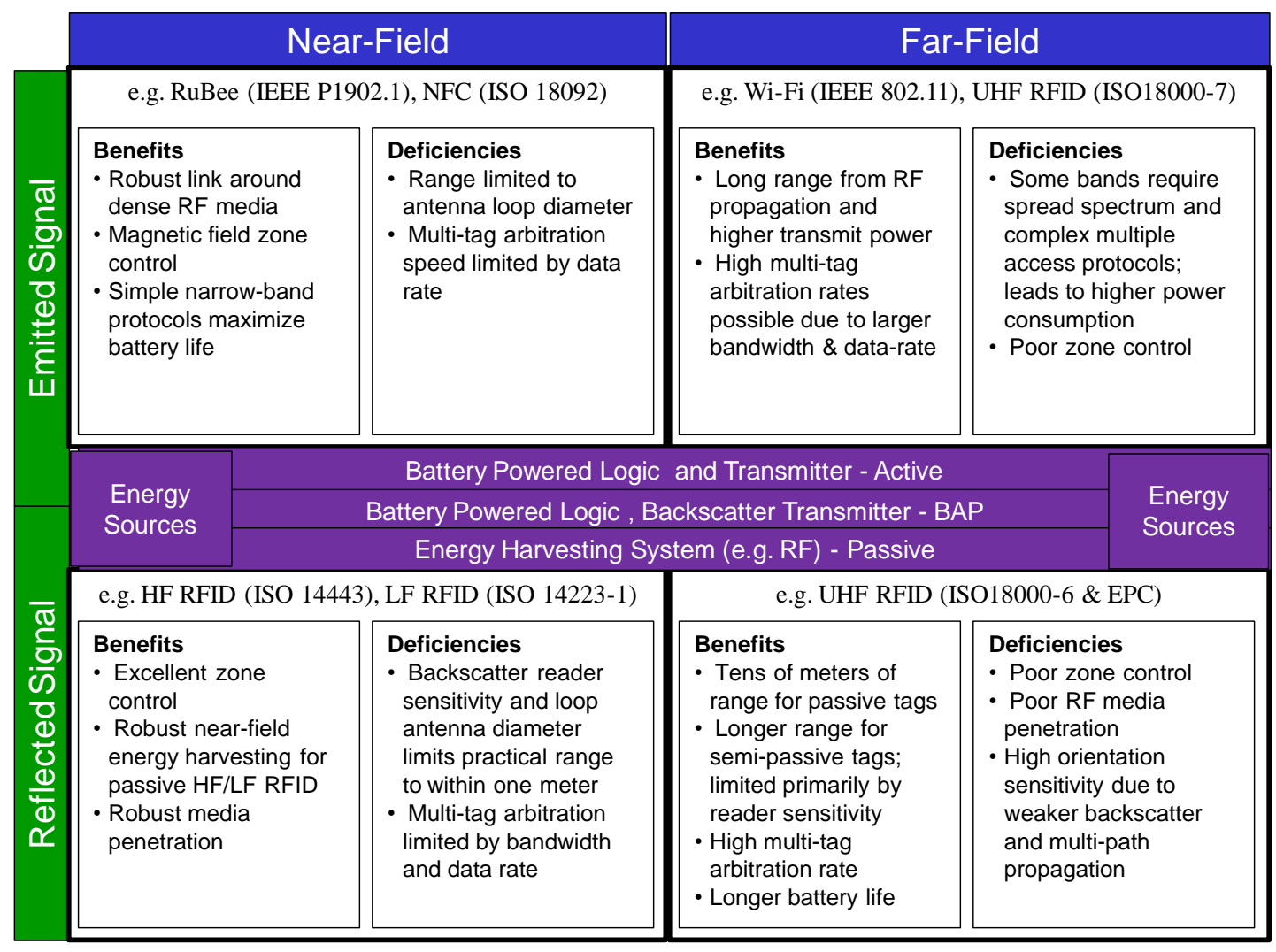

Fig. 3. Operational characteristics of RFID and wireless sensors. 
utilizes tens of megahertz of spectrum and requires spread spectrum, Orthogonal-Frequency-Division-Multiplexing (OFDM), or Ultra-wide Band modulation techniques in order to co-exist and share the spectrum. Depending on the power consumption requirements, any of the technologies in each of these four fundamental categories can harvest energy or utilize a battery. The energy source may be utilized to power only the logic and sensing circuits or to also radiate energy for communications.

A new micro-wireless sensor chip called the Enterprise Dot $^{\mathrm{TM}}$ was recently announced [26]. It is an enhanced dualactive architecture that is uniquely capable of roaming between various near-field and far-field RFID infrastructures using software defined radio techniques. The device is capable of switching the media access (MAC) protocol once a valid preamble is detected from any of the physical (PHY) layers and protocols described in the sections below.

\section{A. Near-Field Backscatter}

A low-frequency (LF) magnetic field ranging from $100 \mathrm{kHz}$ to $150 \mathrm{kHz}$ initiated from a near-field reader or generator. Once the preamble is recognized and the protocol identified, the Enterprise $\operatorname{Dot}^{\mathrm{TM}}$ can respond with synchronous backscatter or load modulation using the appropriate subcarrier modulation type. Such digital modulation procedures include Amplitude Shift Keying (ASK), Frequency Shift Keying (FSK), or Phase-Shift Keying (PSK), and the appropriate symbol encoding scheme, for example, Manchester or Miller. The default protocol is LF activation and LID decoding. Alternatively, one of several proximity access control protocols can be instantiated by vectoring to the appropriate instruction sequence within on-board nonvolatile memory.

\section{B. Far-field Backscatter}

UHF carriers range from $860 \mathrm{MHz}$ to $960 \mathrm{MHz}$ using an ASK modulation. The default protocol is EPC C1G2 (ISO18000-6c). Alternative protocols can be loaded from either internal or external non-volatile memory as needed.

\section{Far-Field Active}

UHF carrier within the $433.92 \mathrm{MHz}$ band. The default protocol is a low-overhead and ultra-low power "Class-IV" bi-directional protocol that is based on noise adaptive multiple access techniques [27]. This transceiver may also be utilized for communications with other wireless devices in a wireless sensor network [28]. Applications include asset protection and security. For example, a belt worn tag may continuously query and monitor for the presence of specific items within a specified communications range.

The Enterprise Dot ${ }^{\mathrm{TM}}$ architecture is shown in Fig. 4. The three integrated PHY radio transceivers utilize separate antennas on a single PCB substrate. An on-board ultra-low power, clock rate agile processor and non-volatile memory can be programmed to support multiple standards for maximum flexibility across applications. Expansion non- volatile memory provides additional storage for additional

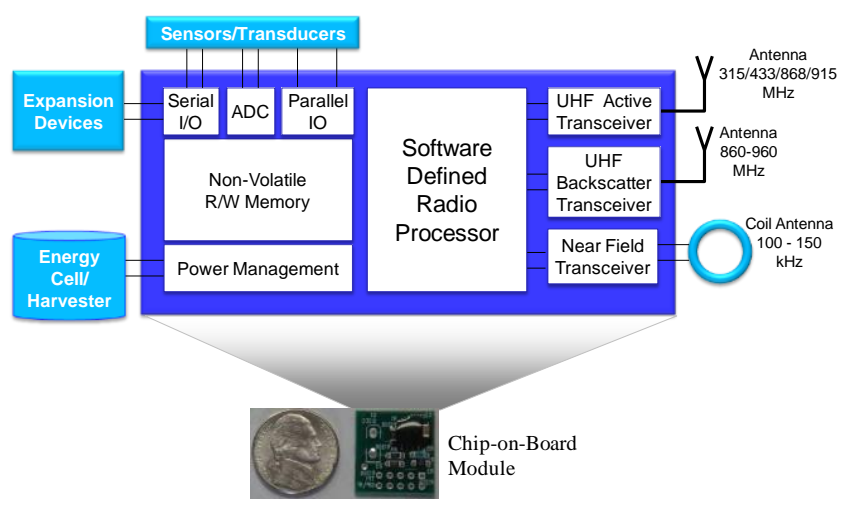

Fig. 4. Architecture of the Enterprise Dot ${ }^{\mathrm{TM}}$.

protocols, application data, and sensor data.

Several options are also available for interfacing to the Enterprise Dot's transducers and other sub-systems using A/D converters. Serial and parallel communications are also supported.

\section{APPLICATION EXAMPLE}

In the United States losses from perishable goods is estimated at $\$ 35$ billion per annum [29]. Much of this loss comes from supply chain mishandling of temperature sensitive items. Perishables such as fresh produce, dairy, meat, cosmetics, and medicine, can be effectively monitored and managed using an automated sense and respond control network. Such a system may be comprised of low-cost RFID sensors that monitor asset location, temperature, fruit ripening processes, and other factors that when perturbed increase risk of spoilage or damage. Once the wireless sensors capture location and condition data, periodic reports or emergency alerts can be sent in real-time to a remote operations center where corrective action can be authorized and taken.

The Enterprise Dot ${ }^{\mathrm{TM}}$, with its combination of near-field localization, and multi-mode far-field communications is capable of roaming across different wireless infrastructures throughout the supply chain. These include LF backscatter for proximity access control, UHF backscatter for passive RFID, and bi-directional UHF links for active RFID communications.

Fig. 5 illustrates three of the key phases in transportation logistics. The first operation involves automatic association of vehicle, driver, and the payload or asset. An Enterprise Dot $^{\mathrm{TM}}$ worn as an access control or identification badge is functionally linked to another Enterprise Dot $^{\mathrm{TM}}$ tag located on the transport vehicle. A near-field control zone ensures accurate co-localization of both tags. This is accomplished when both tags report the same zone identification within seconds of each other and then the association is verified in a database. If the driver-to-vehicle association is authorized for example, the gate can be automatically opened and the 


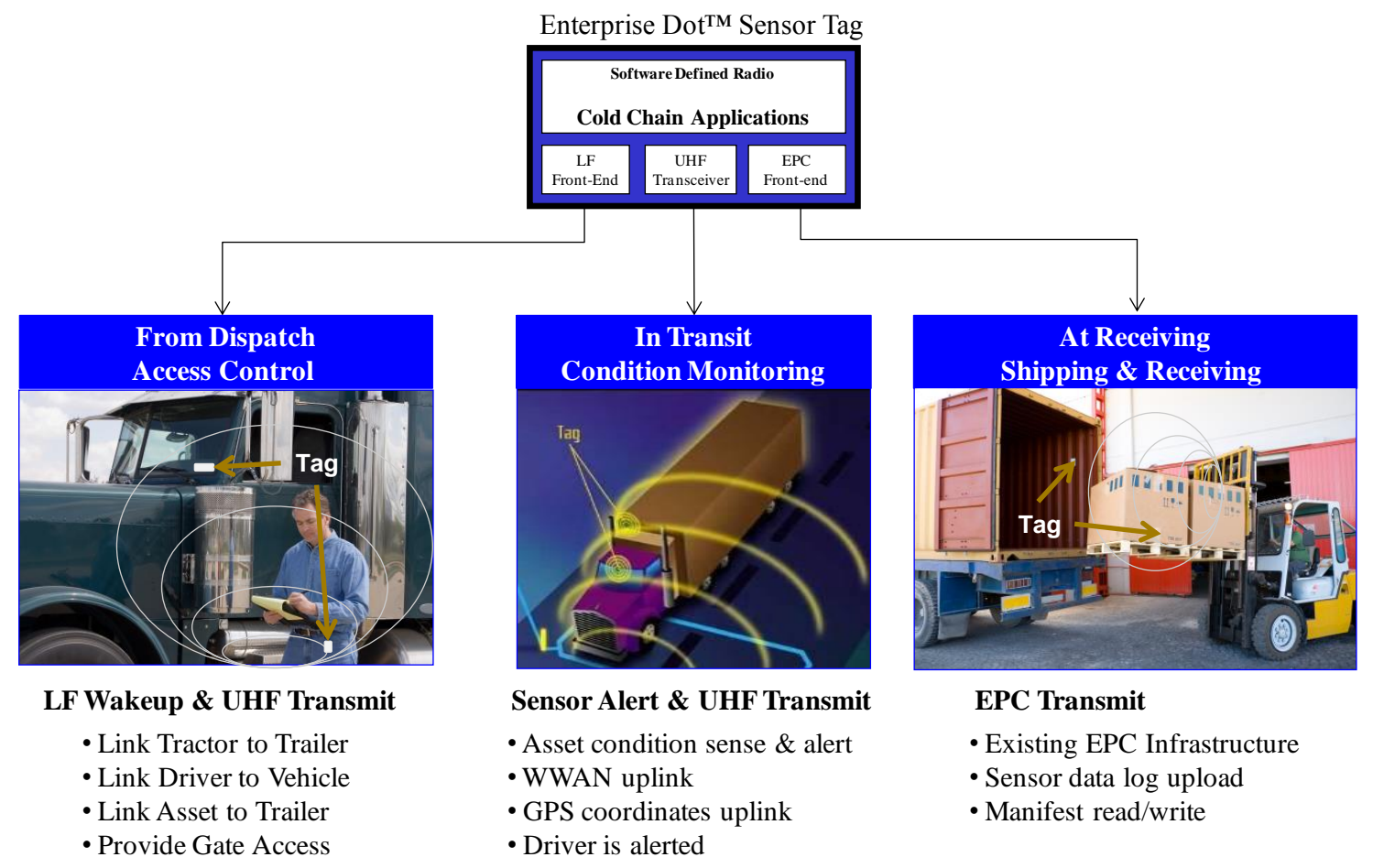

Fig. 5. Key phases of transportation logistics mapped to Enterprise Dot ${ }^{\mathrm{TM}}$ seamless roaming capabilities.

driver can initiate the shipment. One or more personnel identification or asset tags may be linked for additional security or authorization. Linkages such as tractor-to-trailer and asset-to-trailer can be generated and verified automatically as these items move through a control zone.

The second key phase of the logistical process illustrated in Fig. 5 is the journey itself. During this time, if any Enterprise Dot ${ }^{\mathrm{TM}}$ sensor determines that an asset is subject to unsafe limits of either temperature, vibration, or certain gases, then the tag can transmit an alert using the active UHF link. A UHF receiver located within listening distance can time-stamp and log these alerts. Optionally, a wireless wide area network (WWAN) MODEM connected to the UHF receiver can route alert packets to a remote operations center via satellite or cellular network. Alternatively, the logged data may be downloaded via local area network hot spots that might be encountered throughout the journey, such as truck stops and fuel stations.

It is highly probable that the entire shipment can be saved if the alert condition is received in real-time and processed expeditiously. For example, if these alerts are logged but not reported in real-time, then goods may have already spoiled upon arrival, depending on the length of the journey. The supplier can potentially save millions of dollars if instead the shipment is immediately delivered to an alternate customer along the way, even if sold at cost.

The final stage of shipment shown in Fig. 5 is the delivery process. A passive tag reader attached to the forklift can read the Enterprise Dot $^{\mathrm{TM}}$ tag for verification as well as download an entire shipping manifest. The forklift reader can be either a near-field or far-field reader that will also read a tag inside the trailer or shipping container to validate the logical association that exists in a database.

In this application example, we've highlighted each feature of several key communications modes of the Enterprise Dot ${ }^{\mathrm{TM}}$, namely:

1. Near-field localization that also provides high accuracy functional linkages between personnel, vehicles, and assets.

2. Far-field active communications to send real-time, proactive alerts during transport.

3. Near-field or far-field backscatter communications to maintain compliance with existing standards such as $\mathrm{C} 1 \mathrm{G} 2$ passive tag infrastructures.

We also observe that the battery provides a means to log sensor data throughout the journey. This feature is not presently practical with passive RFID tags.

\section{CONCLUSIONS}

The lowest cost RFID tag technology such as passive UHF backscatter is not always the best choice for all applications, even when changes are made to the business process so as to accommodate the technology. The constraints on tag operating characteristics in real-world physical environments and the rigidity of already accepted business processes will eventually limit the scalability of this technology. The most common business process changes involves sorting packages to minimize signal propagation variability, and pre-analyzing packages to find a passive tag location and antenna orientation that minimizes signal losses. These business 
process changes are often expensive and are not scalable across the enterprise.

Although far-field active RFID can improve omnidirectional read rates and minimize the need for business process changes, it introduces other problems such as localization uncertainty, radio interference, and limited battery life. Simply utilizing a mix of active and passive RFID helps the situation but still does not sufficiently address the application requirements due to the combined deficiencies and cost of separately managing disparate technologies.

Dual-active RFID technology combines the benefits of high-accuracy near-field localization and relatively long distance far-field communications. The Enterprise Dot ${ }^{\mathrm{TM}}$ augments the far-field communications capabilities to include UHF backscatter, which allows seamless roaming between various installed active and passive RFID infrastructures.

Dual-active technology also extends battery life by utilizing the near-field generators for system wide ondemand activation. Without near-field activation, a tag must transmit periodically at a high enough rate in order to be tracked within a reasonable degree of accuracy. That is, a tag can be more accurately tracked while moving if it transmits more often. However, the more frequent these transmissions, the higher the power consumption, and the shorter the battery life.

Since the battery is also utilized for powering the sensors during autonomous data logging, the power management algorithms must carefully balance between the need for high accuracy localization, relatively long distance communications, continuous sensing, and air-interface protocol complexity. The Enterprise Dot ${ }^{\mathrm{TM}}$ micro-wireless architecture facilitates this flexibility will undoubtedly trigger an evolution of the next generation of wireless sensors.

\section{REFERENCES}

[1] EPCglobal, Inc., "ЕPC ${ }^{\mathrm{TM}}$ Radio-Frequency Identification protocols Class-1 Generation-2 UHF RFID protocol for communications at 860 $\mathrm{MHz}-960 \mathrm{MHz}$," Specification version 1.1.0, December 17 $7^{\text {th }}, 2005$.

[2] United States Government Accountability Office (GAO), US Border Security US-VISIT, Report No. 07-248, December 2006.

[3] Holt, M., "Does asset tracking live up to the hype?", Health IT World News, March $20^{\text {th }}, 2007$.

[4] Bridgelall, R., "UHF Tags - the answer to the retail supply chain's prayers?," RF Innovations Magazine, Issue 5, August 1999.

[5] Bridgelall, R., "Bluetooth/802.11 protocol adaptation for RFID tags," Proceedings of the $4^{\text {th }}$ European Wireless Conference, Florence, Italy, February $28^{\text {th }}, 2002$.

[6] Hande, A., "Energy harvesting for mobile and wireless electronics", IEEE Dallas Section Meeting, April 2007.

[7] Assad, M. A., "A Real-time laboratory test bed for evaluating localization performance of Wi-Fi RFID technologies", Master's Thesis, Worchester Polytechnic Institute, May $4^{\text {th }}, 2007$.

[8] Leong, K. S., Ng, M. L., Cole, P.H., "Positioning analysis of multiple antennas in dense RFID reader environment", Applications and the Internet Workshops, January $23^{\text {rd }}, 2006$.

[9] IEEE Std. 802.11; Specification is a bases for Wi-Fi' ${ }^{\mathrm{TM}}$.

[10] IEEE Std. 802.15.4; Specification is a bases for ZigBee.
[11] IEEE Std. 802.15.1-2002; Specification is a bases for Bluetooth

[12] IEEE Std. 1902.1; Specification is a bases for Rubee ${ }^{\mathrm{TM}}$.

[13] ISO 18092, Specification is a bases for Near-Field Communications at $13.56 \mathrm{MHz}$.

[14] Clarke, R. H., Twede, D., Tazelaar, J. R., Boyer, K. K., "Radio Frequency Identification (RFID) performance: the effect of tag orientation and package contents", Packaging Technology and Science, Vol. 19, Issue 1, pp. 45-54, November 30 $0^{\text {th }}, 2005$.

[15] Krause, J., Antennas, McGraw-Hill, New York, NY, 1950.

[16] Skolnik, M., Introduction to RADAR Systems, MMcGraw-Hill, New York, NY, 1980

[17] Smith, A. A., Jr., Radio Frequency Principles and Applications, I.E.E.E. Press, N.Y., 1998.

[18] U.S. Code of Federal Regulations (CFR), Title 47, Chapter 1, Part 15: Radio-frequency devices, U.S. Federal Communications Commission.

[19] ISO 18000-7, "Parameters for active air interface communications at $433 \mathrm{MHz}, 2004$.

[20] Starner, T., "Powerful change part 1: batteries and possible alternatives for the mobile market", IEEE Pervasive Computing Mobile and Ubiquitous Systems, Vol. 2, Issue 4, pp. 86-88, October 2003.

[21] US Patent \#7271727, "Dual-frequency radio tag for a radio frequency identification system", September $18^{\text {th }}, 2007$.

[22] US Patent \#6570487, "Distributed tag reader system and method", May 2003.

[23] US Patent \#6294953, "High sensitivity demodulation for a radio tag and method", September 2001.

[24] Higgins, L. N., Cairney, T., "RFID opportunities and risks", Journal of Corporate Accounting and Finance, Vol. 17, Issue 5, pp. 51-57, June $19^{\text {th }}, 2006$.

[25] US Patent Publication \#20070205896, "System and method for determining location, directionality, and velocity of RFID tags," September $6^{\text {th }}, 2007$.

[26] Axcess International Inc., "Dot" ${ }^{\mathrm{TM}}$ micro-wireless technology for business activity monitoring," Press Release, November 2007.

[27] US Patent \#6034603, "Radio tag system and method with improved tag interference avoidance," March $7^{\text {th }}, 2000$.

[28] US Patent \#7005985, "Radio frequency identification system and method", February $28^{\text {th }}, 2006$.

[29] Deloitte \& Touche USA LLC, "Intelligent cold chain: capturing the value of pervasive computing for supply chain transformation," December 2006. 